\title{
Electrothermal flows generated by alternating and rotating electric fields in microsystems
}

\author{
By A. GONZÁLEZ1, A. RAMOS ${ }^{2} \dagger$, H. MORGAN \\ N. G. GREEN AND A. CASTELLANOS \\ ${ }^{1}$ Departamento Física Aplicada III, E.S.I. University of Seville, Camino de los Descubrimientos \\ s/n, 41092 Sevilla, Spain \\ ${ }^{2}$ Departamento Electrónica y Electromagnetismo, University of Seville, Avda. Reina Mercedes \\ s/n, 41012 Sevilla, Spain \\ ${ }^{3}$ School of Electronics and Computer Science, University of Southampton, SO17 1BJ, UK
}

(Received 27 May 2005 and in revised form 2 March 2006)

Electrothermal motion in an aqueous solution arises from the action of an electric field on inhomogeneities in the liquid induced by temperature gradients. The temperature field can be produced by the applied electric field through Joule heating, or caused by external sources, such as strong illumination. Electrothermal flows in microsystems are usually observed at applied signal frequencies around $1 \mathrm{MHz}$ and voltages around $10 \mathrm{~V}$. In this work, we present self-similar solutions for the motion of an aqueous solution in a constant temperature gradient placed on top of: (a) two coplanar electrodes subjected to an a.c. potential difference, and $(b)$ four coplanar electrodes subjected to a four-phase a.c. signal, generating a rotating field. The first case produces two-dimensional rolls whereas the second case produces a liquid whirl. Finally, we present experimental results of electrothermal liquid flows generated by alternating and rotating electric fields under strong illumination, and these experiments are compared to the analytical solutions. The induced rotating flow could be used in the mixing of analytes and of liquids in microsystems.

\section{Introduction}

In microsystems, a.c. electric fields can interact with a liquid through several mechanisms (Castellanos et al. 2003), ranging from bulk effects such as electrothermal effects and natural convection (Müller et al. 1993; Arnold 2001) to surface effects such as ac electro-osmosis (Ramos et al. 1999; Ajdari 2000; Bazant \& Squires 2004). Flows driven by electrothermal forces are often present in the dielectrophoretic manipulation of colloidal particles in microsystems (Müller et al. 1996; Ramos et al. 1998; Green et al. 2000, 2001). Electrothermal fluid flow is due to the action of an electric field on thermally induced gradients of conductivity and permittivity in the fluid (Melcher \& Firebaugh 1967; Melcher 1981). The gradients of temperature can be produced by external sources, such as strong illumination (Green et al. 2000), or caused by the applied electric field through Joule heating (Müller et al. 1993; Gimsa, Eppmann \& Prüger 1997; Wang, Sigurdson \& Meinhart 2005). Observations and estimates show that electrothermal effects are important in microsystems for frequencies of the order of $1 \mathrm{MHz}$ and voltages of the order

$\dagger$ Author to whom correspondence should be addressed: ramos@us.es. 


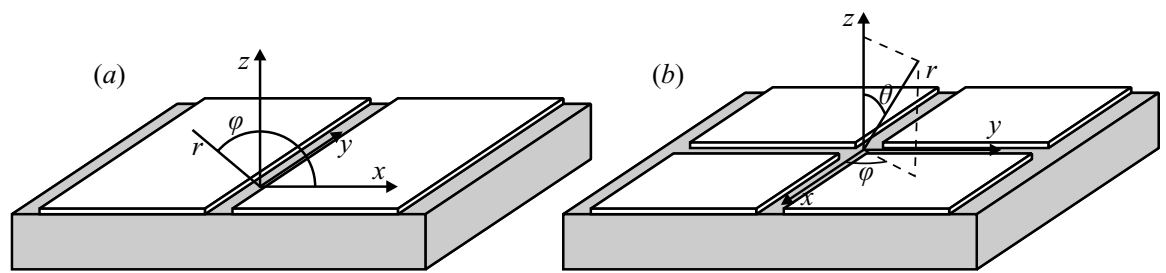

FIGURE 1. Diagrams showing the coordinate systems for: $(a)$ the two coplanar electrodes and (b) the four coplanar electrodes.

of $10 \mathrm{~V}$ (Green et al. 2001; Castellanos et al. 2003). These frequencies are much greater than typical frequencies of electrode polarization. For these frequencies, electrokinetic flows such as a.c. electro-osmosis are almost certainly negligible since the charges have no time to accumulate in the diffuse double layer (Ramos et al. 1999). In this work, we will consider that the applied signals have frequencies in this regime, i.e. $\omega \gg 1 / R_{b} C_{D L}$, where $R_{b}$ is the resistance of the bulk electrolyte between electrodes and $C_{D L}$ the capacitance of the electrode/electrolyte double layer.

In this paper, we present some self-similar solutions for the fluid flow induced by a combination of electric fields and externally imposed gradients of temperature in microelectrode structures. First, we consider the two-dimensional motion of an aqueous solution placed on top of two coplanar, parallel electrodes subjected to an a.c. potential difference when there is either a vertical or a horizontal gradient of temperature. This geometry is shown in figure 1(a). Self-similar solutions for the electric field and liquid motion are obtained. These solutions are relevant to some fluid flows observed under strong illumination (Green et al. 2000).

Secondly, we study the three-dimensional problem of an aqueous solution lying on four coplanar electrodes placed in each quadrant (see figure $1 b$ ). The electrodes are driven by a four-phase a.c. signal and this generates a rotating electric field. Previously studied two-dimensional configurations are used to solve the electrical three-dimensional problem analytically. Then, the solution for the liquid flow is obtained and it is shown that this produces a rotation of the liquid. Such a system could be used in the design of rotatory micro-pumps and for local mixing in microfluidics. Also, these results are relevant to electrorotation experiments when combined with laser tweezers, where electrothermal flow rotation has been observed (Schnelle et al. 2000).

Finally, we present experimental results of fluid flow and compare them with both the two-dimensional and three-dimensional analytical self-similar solutions. In the experiments, we have used simple electrode designs consisting of two or four coplanar electrodes mounted on a glass substrate. Aqueous solutions of Potassium chloride $(\mathrm{KCl})$ were placed on top of the electrodes and subjected to the electric fields. The temperature field was imposed using the light from a microscope (Green et al. 2000, 2001). A clear indication of heating under the strong illumination from the microscope was the observation of natural convection, in the absence of electric fields, for devices with heights of the order of $1 \mathrm{~mm}$. For devices with smaller heights, gravity could not overcome the viscous friction, and fluid flow was not observed in the absence of electric fields. The combination of a.c. electric fields and heating by light at localized points in a fluid could be of interest for the handling of liquids in lab-on-a-chip technology. 


\section{Formulation of the problem}

The mathematical problem involves a coupled system of electrical, mechanical and thermodynamical equations. The electric field moves the liquid, and this motion in turn convects charge and heat. Nevertheless, the low velocities involved in microsystems mean that these convection terms can be neglected, and therefore, the electrical and thermodynamical problems can be solved independently with the solution then inserted into the mechanical problem (Ramos et al. 1998).

To determine whether the temperature field is independent of the velocity field, the Péclet number must be calculated, given by $v l / \kappa$ ( $v$, typical velocity; $l$, typical system length; $\kappa$, thermal diffusivity). The Péclet number for microsystems is typically very small, showing that heat convection is small compared to heat diffusion (Castellanos et al. 2003).

In the bulk electrolyte, the electrical current $j$ is given very approximately by Ohm's law, $\boldsymbol{j}=\sigma \boldsymbol{E}$ (Levich 1962). For a binary monovalent electrolyte at rest, the current density can be expressed as

$$
\boldsymbol{j}=e\left(n_{+} \mu_{+}+n_{-} \mu_{-}\right) \boldsymbol{E}-e\left(D_{+} \nabla n_{+}-D_{-} \nabla n_{-}\right)-e\left(D_{+}^{T} n_{+}-D_{-}^{T} n_{-}\right) \nabla T,
$$

with $e$ the absolute value of electronic charge, $\mu, D, D^{T}, n_{+}$and $n_{-}$, the mobility, diffusion coefficient, thermodiffusion coefficient and number densities of positive and negative ions. For typical electric fields of interest in microsystems, the electromigration current dominates. In effect, the ratio between diffusion and electrical drift is of the order of $D_{+} / \mu_{+} E l=k_{B} T / e E l$ (Chen et al. 2005), and the ratio between thermodiffusion and electrical drift is of the order of $\Delta T D_{+}^{T} / \mu_{+} E l \sim$ $\Delta T D_{+} / T \mu_{+} E l=k_{B} \Delta T / e E l$ (Putnam \& Cahill 2005). Both ratios are much less than one $\left(k_{B} T / e \approx 0.025 \mathrm{~V}, E l \sim 5 \mathrm{~V}\right)$. Here $k_{B}$ is Boltzmann's constant, $T$ is the absolute temperature and $l$ is a typical distance. In addition, Gauss' law suggests that the liquid bulk is quasi-electroneutral on the micrometre length scale. The relative difference in ion number densities $\left(n_{+}-n_{-}\right) / n_{+}=\nabla \cdot(\varepsilon \boldsymbol{E}) / e n_{+}$is of the order of the parameter $\Lambda=\varepsilon E / e l n_{+}$(Saville 1997; Castellanos et al. 2003), which is very small for typical values in microsystems. The conductivity in Ohm's law is then given by $\sigma=e\left(\mu_{+}+\mu_{-}\right) n_{0}$, with $n_{0}$ the unperturbed ion density.

In this work, we assume that the origin of conductivity gradients is due to the fact that ionic mobilities depend on temperature. The conductivity is then a given function of temperature $\sigma=\sigma(T)$. We do not take into consideration gradients of concentration, as in Chen et al. (2005). Gradients of concentration can appear at the electrodes owing to concentration polarization. For applied a.c. voltages of high enough frequencies $\left(\omega \gg 1 / R_{b} C_{D L}\right)$, negligible charge is accumulated at the double layer and no concentration polarization is expected. This ' $\mathrm{RC}$ time' of a bulk resistor in series with a double-layer capacitor is of the order of $(\varepsilon / \sigma)\left(l / \lambda_{D}\right)$, where $\lambda_{D}$ is the Debye length (Ramos et al. 1999; Bazant et al. 2004). We consider a.c. signals with angular frequencies that are $\omega \gg \sigma \lambda_{D} / \varepsilon l\left(f=\omega / 2 \pi \gg 1 \mathrm{kHz}\right.$ for $\sigma \sim 10^{-3} \mathrm{~S} \mathrm{~m}^{-1}$ and $l \sim 10^{-5} \mathrm{~m}$ ). In addition, the diffusion equation that governs the conductivity (Bazant et al. 2004; Chen et al. 2005) gives a typical diffusion length of the order of $\sqrt{D / \omega}$, which is very small, of the order of the Debye length for frequencies around $\sigma / \varepsilon$. Therefore, we do not expect gradients of concentration in the bulk.

The liquid conductivity and permittivity are functions of temperature. The temperature field is a function of the electric field due to Joule heating and, therefore, the electrical equations are strongly coupled to the temperature equation (Castellanos 2003). Typical increments in temperature caused by Joule heating are compared to 
imposed temperature fields in Green et al. (2001); Castellanos et al. (2003). It was shown that for applied voltages smaller than or equal to $10 \mathrm{~V}$, and conductivities less than $0.03 \mathrm{~S} \mathrm{~m}^{-1}$, Joule heating is insufficient to generate the gradients of temperature that account for observed flow velocities (which are of the order of $50 \mu \mathrm{m} \mathrm{s}^{-1}$ ). Wang et al. (2005) have measured fluid velocities around $100 \mu \mathrm{m} \mathrm{s}^{-1}$ for electrothermal flow induced by Joule heating at voltage amplitudes of $10-15 \mathrm{~V}$, but in a fluid of conductivity $\sigma=0.056 \mathrm{~S} \mathrm{~m}^{-1}$. For the conductivity we analyse in the experimental section, the same voltages would generate fluid velocities around $5 \mu \mathrm{m} \mathrm{s}^{-1}$ or less. In this paper, we consider only externally imposed temperature gradients, which are independent of the electric field.

\subsection{Electrical equations}

At low frequencies $(<100 \mathrm{MHz})$ and because the magnetic effects are small, the electromagnetic equations reduce to the quasi-electrostatic limit (Haus \& Melcher 1989). In addition, the convection current can be neglected when compared to the ohmic current (Castellanos et al. 2003). The electrical Reynolds number is defined as $\varepsilon v / l \sigma$ (Melcher \& Taylor 1969), where $\varepsilon$ and $\sigma$ are the electrical permittivity and conductivity of the fluid, and $v$ and $l$ are the typical velocity and length in the system. For microsystems, this number is much less than one, implying that ohmic currents dominate. Assuming that $\sigma$ and $\varepsilon$ are independent of time, the equations that govern the electric fields are

$$
\begin{gathered}
\nabla \cdot(\varepsilon \boldsymbol{E})=\rho, \\
\nabla \cdot(\sigma \boldsymbol{E})=-\frac{\partial \rho}{\partial t}, \\
\nabla \times \boldsymbol{E}=0 .
\end{gathered}
$$

As the applied voltage is an a.c. signal of angular frequency $\omega$, we use complex amplitudes for the electric field $\boldsymbol{E}(t)=\operatorname{Re}\left(\boldsymbol{E} \mathrm{e}^{\mathrm{i} \omega t}\right)$, where $\operatorname{Re}(\ldots)$ indicates the real part of $(.$.$) , and combine the equations to give$

$$
\nabla \cdot((\sigma+\mathrm{i} \omega \varepsilon) \boldsymbol{E})=0, \quad \nabla \times \boldsymbol{E}=0 .
$$

The electric field can be written as the gradient of an electric potential. The boundary conditions for this potential are given by the voltage applied to the electrodes, $V_{s}(x, y)$ at the plane $z=0$

$$
\phi=V_{s}(x, y) \quad(z=0),
$$

with the origin of potential located at infinity

$$
\phi \rightarrow 0 \quad(z \rightarrow \infty)
$$

In (6), we have assumed that the frequency $\omega$ is high, so that the voltage across the double layer is negligible.

\subsection{Mechanical equations}

After calculating the electric field, the electrical volume force density can be determined. Ignoring the electrostriction term (that can be incorporated into the pressure and omitted from the calculations), the instantaneous electrical force density is given by the sum of a Coulomb term and a dielectric term (Melcher \& Taylor 1969)

$$
\boldsymbol{f}=\rho \boldsymbol{E}-\frac{1}{2} E^{2} \nabla \varepsilon
$$


Because $\rho$ and $\boldsymbol{E}$ are oscillating at a frequency $\omega$, the volume force is composed of a steady term and an oscillating force, of frequency $2 \omega$. This second term would produce a small-amplitude rapid oscillation of the liquid, which is impossible to observe in practice. The steady component of the force produces a continuous motion that is easily observable. This force can be calculated as the time-averaged value of the electrical volume force density acting on the fluid (Ramos et al. 1998)

$$
\langle\boldsymbol{f}\rangle=\frac{1}{2} \operatorname{Re}\left(\rho \boldsymbol{E}^{*}\right)-\frac{1}{4} \boldsymbol{E} \cdot \boldsymbol{E}^{*} \nabla \epsilon,
$$

where * indicates complex conjugate.

This average force is inserted into the Navier-Stokes equations to calculate the liquid motion. In the low-Reynolds-number regime (valid for microsystems), the equations for the time-averaged velocity and pressure are

$$
\nabla \cdot v=0, \quad-\nabla p+\eta \nabla^{2} \boldsymbol{v}+\langle\boldsymbol{f}\rangle=0
$$

Here we have neglected the buoyancy force. For microsystems with typical lengths smaller than $300 \mu \mathrm{m}$, the buoyancy force is small compared to the electrical force (Ramos et al. 1998; Castellanos et al. 2003). We have also neglected variations of liquid viscosity with temperature in the mechanical equations, as is usually done in the Boussinesq approximation (Tritton 1977). The boundary condition for the liquid velocity at the electrode plane is simply $\boldsymbol{v}=0$. We have assumed that the frequency is high enough so that negligible charge is accumulated in the diffuse double layer and, therefore, possible electrokinetic slip velocities are also negligible.

\subsection{Weak temperature gradient}

Even for applied temperature gradients, the spatial dependence of the field may be complicated. We assume that the permittivity and conductivity changes with temperature are small. In this way, we can expand $\varepsilon$ and $\sigma$ around a reference temperature as

$$
\varepsilon(T)=\varepsilon^{0}\left(1+\alpha\left(T-T_{0}\right)\right) \quad \sigma(T)=\sigma^{0}\left(1+\beta\left(T-T_{0}\right)\right),
$$

where in the right-hand side of each equation, $\sigma^{0}, \varepsilon^{0}, \alpha$ and $\beta$ are the quantities and their relative derivatives at $T=T_{0}$. For aqueous solutions at $T_{0}=20^{\circ} \mathrm{C}, \alpha=$ $(\partial \varepsilon / \partial T) / \varepsilon \approx-0.0046 \mathrm{~K}^{-1}$ and $\beta=(\partial \sigma / \partial T) / \sigma \approx 0.020 \mathrm{~K}^{-1}$ (from CRC Handbook of Chemistry and Physics).

In the same approximation, the electric field can be written as $\boldsymbol{E}=\boldsymbol{E}_{0}+\boldsymbol{E}_{1}$, where $\boldsymbol{E}_{0}$ is the electric field for a spatially constant temperature, $T=T_{0}$, and $\left|\boldsymbol{E}_{1}\right| \ll\left|\boldsymbol{E}_{0}\right|$. Substituting in the equations for the field, we have, at the lowest order

$$
\nabla \cdot \boldsymbol{E}_{0}=0, \quad \nabla \times \boldsymbol{E}_{0}=0, \quad \rho_{0}=0,
$$

so that the electric potential at this order satisfies Laplace's equation, $\nabla^{2} \phi=0$, with boundary conditions given by the applied voltage, $V_{s}$, at the plane $z=0$.

\subsection{Volume force}

The first non-vanishing term in the expansion of the electrical force requires the first-order charge density $\rho_{1}$, whose complex amplitude can be obtained from the zero-order field (Ramos et al. 1998; Castellanos et al. 2003). Taking into account (2) and (5), the complex amplitude $\rho_{1}$ is

$$
\rho_{1}=\nabla \varepsilon \cdot \boldsymbol{E}_{0}+\varepsilon^{0} \nabla \cdot \boldsymbol{E}_{1}=\nabla \varepsilon \cdot \boldsymbol{E}_{0}-\varepsilon^{0} \frac{\boldsymbol{E}_{0} \cdot \nabla(\sigma+\mathrm{i} \omega \varepsilon)}{\sigma^{0}+\mathrm{i} \omega \varepsilon^{0}}
$$


and from $\nabla \varepsilon=\varepsilon^{0} \alpha \nabla T$ and $\nabla \sigma=\sigma^{0} \beta \nabla T$, we obtain

$$
\rho_{1}=\frac{\varepsilon^{0}(\alpha-\beta)}{1+\mathrm{i} \omega \tau}\left(\nabla T \cdot \boldsymbol{E}_{0}\right)
$$

with $\tau=\varepsilon^{0} / \sigma^{0}$, the charge relaxation time of the liquid. Substituting in the timeaveraged volume force, (9), gives:

$$
\langle\boldsymbol{f}\rangle=\frac{1}{2} \operatorname{Re}\left(\frac{\varepsilon^{0}(\alpha-\beta)}{1+\mathrm{i} \omega \tau}\left(\nabla T \cdot \boldsymbol{E}_{0}\right) \boldsymbol{E}_{0}^{*}\right)-\frac{\varepsilon^{0}}{4} \alpha \boldsymbol{E}_{0} \cdot \boldsymbol{E}_{0}^{*} \nabla T .
$$

\section{Two-dimensional flows}

We consider first the geometry shown in figure 1(a), which consists of two coplanar electrodes separated by a small gap.

Approximating the electrodes as half-planes separated by an infinitesimal gap, the boundary conditions for the potential at $z=0$ are

$$
\phi(z=0)=V_{s}=\frac{1}{2} V_{0} \operatorname{sgn}(x), \quad \operatorname{sgn}(x)= \begin{cases}+1 & x>0 \\ -1 & x<0 .\end{cases}
$$

This problem allows a self-similar solution, independent of $r$, given in polar coordinates by

$$
\phi(r, \varphi)=\frac{V_{0}}{\pi}\left(\frac{\pi}{2}-\varphi\right), \quad \boldsymbol{E}_{0}(r, \varphi)=\frac{V_{0}}{\pi r} \boldsymbol{u}_{\varphi} .
$$

The field lines describe half-circles from one electrode to the other. This solution describes approximately the behaviour of a real system if the gap is much narrower than the electrode width.

Using Cartesian coordinates, the voltage and the field can be written as

$$
\phi=\frac{V_{0}}{\pi} \arctan \left(\frac{x}{y}\right), \quad \boldsymbol{E}_{0}=\frac{V_{0}}{\pi}\left(\frac{-y \boldsymbol{u}_{x}+x \boldsymbol{u}_{y}}{x^{2}+y^{2}}\right) .
$$

\subsection{Vertical gradient}

Assume an imposed uniform vertical gradient of temperature, that can be written as $\nabla T=T^{\prime} \boldsymbol{u}_{z}$. Substituting in (15), we obtain the first-order electrical body force

$$
\langle\boldsymbol{f}\rangle=\frac{\varepsilon^{0}}{2}\left(\frac{V_{0}}{\pi r}\right)^{2} T^{\prime}\left[\left(\frac{\alpha-\beta}{1+(\omega \tau)^{2}}-\frac{\alpha}{2}\right) \cos \varphi \boldsymbol{u}_{\varphi}-\frac{\alpha}{2} \sin \varphi \boldsymbol{u}_{r}\right] .
$$

The $r^{-2}$ radial dependence of $\langle\boldsymbol{f}\rangle$ enables self-similar solutions to be found for the pressure and liquid velocity. These solutions are expressed as the product of a certain power of $r$ multiplied by a function of the angle $\varphi$, i.e. $r^{n} F(\varphi)$. A simple scale analysis gives the appropriate powers from the Stokes equation: $p \sim r^{-1}, v \sim r^{0}, f \sim r^{-2}$. From this balance, we propose the functional forms

$$
p(r, \varphi)=\frac{P(\varphi)}{r}, \quad \boldsymbol{v}=\left(v_{r}(\varphi) \boldsymbol{u}_{r}+v_{\varphi}(\varphi) \boldsymbol{u}_{\varphi}\right) .
$$

The incompressibility condition provides a relation between the radial and azimuthal components

$$
v_{r}=-\frac{\mathrm{d} v_{\varphi}}{\mathrm{d} \varphi} .
$$


Substituting in the Navier-Stokes equation and eliminating the pressure, we obtain a fourth-order equation for the azimuthal velocity

$$
\frac{\mathrm{d}^{4} v_{\varphi}}{\mathrm{d} \varphi^{4}}+2 \frac{\mathrm{d}^{2} v_{\varphi}}{\mathrm{d} \varphi^{2}}+v_{\varphi}=-C \cos \varphi
$$

where

$$
C=\frac{\varepsilon^{0} V_{0}^{2} T^{\prime}}{2 \pi^{2} \eta} \frac{\alpha(\omega \tau)^{2}+\beta}{1+(\omega \tau)^{2}}
$$

Since $\alpha<0$ and $\beta>0$, the constant $C$ changes sign at a characteristic frequency given by

$$
\omega_{c}=\frac{1}{\tau} \sqrt{\frac{\beta}{-\alpha}}
$$

which gives approximately $\omega_{c} \approx 2.1 / \tau$ for electrolytes. The general solution for $v_{\varphi}$ is:

$$
v_{\varphi}=\frac{1}{8} C \varphi^{2} \cos \varphi+A_{1} \cos \varphi+A_{2} \varphi \cos \varphi+B_{1} \sin \varphi+B_{2} \varphi \sin \varphi .
$$

The boundary conditions for zero velocity at $\varphi=0$ and $\pi$ require

$$
v_{\varphi}=0 \quad \text { at } \varphi=0, \pi, \quad \frac{\mathrm{d} v_{\varphi}}{\mathrm{d} \varphi}=0 \quad \text { at } \quad \varphi=0, \pi .
$$

This gives the solution for $v_{\varphi}$ as

$$
v_{\varphi}=-\frac{1}{8} C\left(\left(\pi \varphi-\varphi^{2}\right) \cos \varphi+(2 \varphi-\pi) \sin \varphi\right),
$$

and from here, the radial component can be obtained,

$$
v_{r}=-\frac{\mathrm{d} v_{\varphi}}{\mathrm{d} \varphi}=\frac{1}{8} C\left(2-\pi \varphi+\varphi^{2}\right) \sin \varphi .
$$

For this particular solution, the fluid flow changes direction at the characteristic frequency $\omega_{c}$, equation (24). For the general case, there is a transition of fluid flow behaviour around $\omega_{c}$, corresponding to fluid flow driven by two different forces: the Coulomb term (for $\omega \ll \omega_{c}$ ) and the dielectric term (for $\omega \gg \omega_{c}$ ). Furthermore, the difference in magnitude between the Coulomb force and the dielectric force implies that the velocity amplitude at low frequencies is $\beta /|\alpha| \sim 5$ times greater than the velocity amplitude at high frequencies.

In this self-similar solution, the velocity components do not depend on the distance to the origin $r$. Figure 2(a) shows a contour plot of a streamfunction, defined by the relations

$$
v_{r}=-\frac{1}{r} \frac{\partial \psi}{\partial \varphi}, \quad v_{\varphi}=\frac{\partial \psi}{\partial r} .
$$

The arrows indicate the direction of the fluid flow when the temperature decreases with height and the frequency is less than $\omega_{c}$ as defined by (24). For $\omega>\omega_{c}$, the flow changes direction. The pattern of streamlines is very close to that given in Green et al. (2001) for a similar numerical problem in the neighbourhood of $r=0$ where the self-similar solution is expected to be valid.

Figure 2(b) shows a plot of the modulus of the fluid velocity (scaled with $C$ ) for the same system. The maximum absolute value of $v_{r}$ occurs at $\varphi=\pi / 2$, where $v_{r}=-0.0584251 C$. The maximum absolute value of $v_{\varphi}$ is at $\varphi_{1}=0.887129$ and $\varphi_{2}=\pi-\varphi_{1}$ where $v_{\varphi}\left(\varphi_{1}\right)=0.025405 C$ and $v_{\varphi}\left(\varphi_{2}\right)=-0.025405 C$. These values of maximum velocities compare well with the numerical results presented in Green et al. 


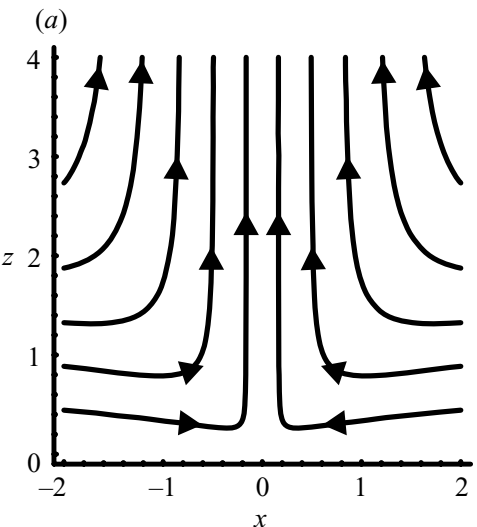

(b)

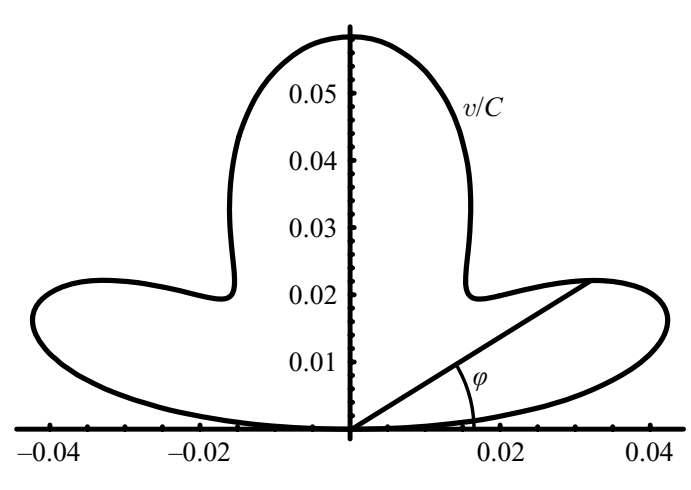

FIGURE 2. (a) Streamlines for a vertically imposed temperature gradient. The arrows indicate the direction of the fluid flow when the temperature decreases with height and the frequency is less than $\omega_{c}$. $(b)$ Liquid speed as a function of angle $\varphi$ for a vertically imposed temperature gradient (polar curve).

(2001) for a system of two coplanar electrodes heated by light. In this work, the numerically calculated thermal field shows an almost vertical gradient of temperature over the electrodes. Using the values specified in Green et al. (2001), $T^{\prime}=0.021 \mathrm{~K}^{\prime} \mathrm{m}^{-1}$, $V_{0}=10 \mathrm{~V}$ and $\omega \ll \omega_{c}$, the maximum radial velocity from the analytical solution is $87.0 \mu \mathrm{ms}^{-1}$, compared to $80 \mu \mathrm{ms}^{-1}$ obtained from numerical work using finite-element modelling.

\subsection{Horizontal gradient}

Assume now an imposed horizontal gradient of temperature in the $x$ direction, $\nabla T=$ $T^{\prime} \boldsymbol{u}_{x}$. In polar coordinates, this is

$$
T(r, \varphi)=T_{0}+T^{\prime} r \cos \varphi,
$$

where the temperature at the plane $\varphi=\pi / 2$ (or $x=0$ ) is $T_{0}$.

Assuming again that the permittivity and conductivity increments with temperature are small enough, we can write the electrical body force as:

$$
\langle\boldsymbol{f}\rangle=-\frac{\varepsilon^{0}}{2}\left(\frac{V_{0}}{\pi r}\right)^{2} T^{\prime}\left[\left(\frac{\alpha-\beta}{1+(\omega \tau)^{2}}-\frac{\alpha}{2}\right) \sin \varphi \boldsymbol{u}_{\varphi}+\frac{\alpha}{2} \cos \varphi \boldsymbol{u}_{r}\right],
$$

where $\alpha$ and $\beta$ have the same meaning as before.

Since the body force tends again as $r^{-2}$, we can propose self-similar solutions as previously. The resulting equation for the azimuthal velocity is now

$$
\frac{\mathrm{d}^{4} v_{\varphi}}{\mathrm{d} \varphi^{4}}+2 \frac{\mathrm{d}^{2} v_{\varphi}}{\mathrm{d} \varphi^{2}}+v_{\varphi}=C \sin \varphi
$$

where $C$ is the same constant as given in (23).

The solution for $\varphi$ that satisfies the boundary conditions for zero velocity at $\varphi=0$ and $\pi$ is

$$
v_{\varphi}=\frac{1}{8} C\left(\pi \varphi-\varphi^{2}\right) \sin \varphi .
$$

The expression for the radial component is

$$
v_{r}=-\frac{1}{2} C\left((\pi-2 \varphi) \sin \varphi+\left(\pi \varphi-\varphi^{2}\right) \cos \varphi\right) .
$$


(a)

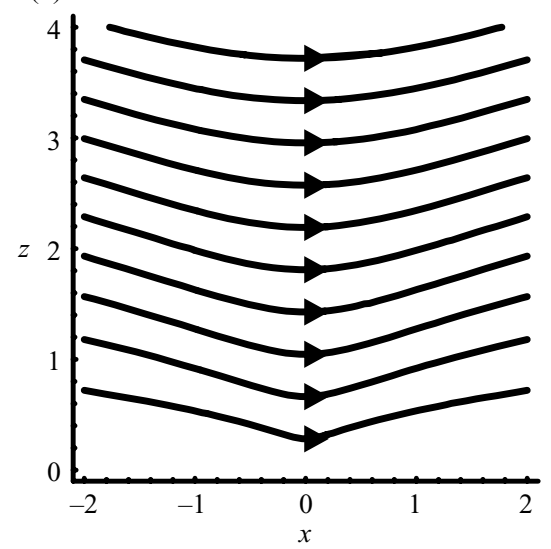

(b)

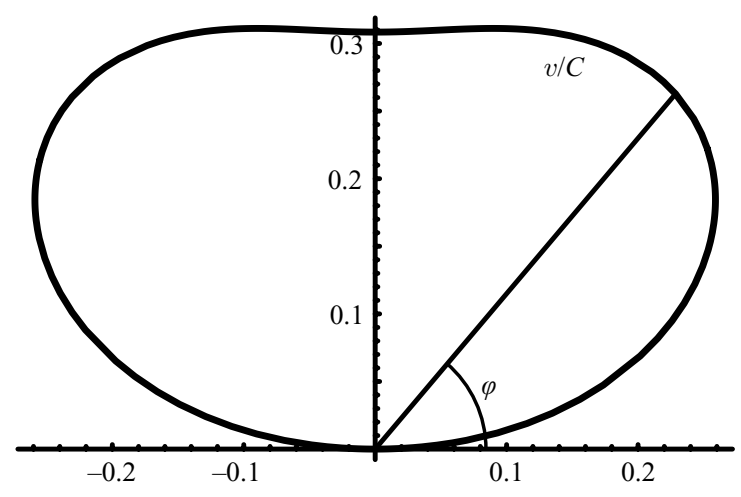

FigURE 3. (a) Streamlines for a horizontally imposed temperature gradient. The arrows indicate the direction of the fluid flow when the temperature decreases with $x$ (to the right in the figure) and $\omega<\omega_{c}$. (b) Liquid speed as a function of angle $\varphi$ for a horizontally imposed temperature gradient (polar curve).

Figure 3(a) shows a contour plot of the corresponding streamfunction. The arrows indicate the direction of the fluid flow when the temperature decreases with $x$ (to the right in the figure) and $\omega<\omega_{c}$. As before, for $\omega>\omega_{c}$ the flow changes direction. Figure $3(b)$ shows the modulus of the velocity for the same system. This solution is relevant to experiments where a difference in temperature is established between coplanar electrodes, producing a horizontal gradient (Green et al. 2000, 2001). Since unidirectional motion is obtained, this configuration could be used as the basis for a micro-pump employing only a single a.c. signal. For example, an array of interdigitated electrodes with a modulated temperature field could be used. An advantage of this type of pump is that for saline solutions, the maximum flow velocity is almost independent of the conductivity of the solution. In effect, the peak velocity is obtained at frequencies $\omega \ll \omega_{c}$ and it is proportional to $\beta=(\mathrm{d} \sigma / \mathrm{d} T) / \sigma$. In order to avoid complications with double-layer effects, the frequency of the applied signal should be increased linearly with conductivity, keeping $\omega \ll \omega_{c}$.

The maximum absolute value of $v_{\varphi}$ is obtained at $\varphi=\pi / 2$, where $v_{\varphi}=0.308425 \mathrm{C}$. The maximum absolute value of $v_{r}$ is at $\varphi_{1}=0.729827$ and $\varphi_{2}=\pi-\varphi_{1}$ where $v_{r}\left(\varphi_{1}\right)=-0.304156 C$ and $v_{r}\left(\varphi_{2}\right)=0.304156 C$.

\section{Three-dimensional fluid rotation}

Extending the analysis to three dimensions, we consider a system of four electrodes placed at each quadrant, with small gaps in between, and with a rotating-phase voltage applied (see figure $1 b$ ).

In this system, the electric field has a net rotation around the $z$-axis. The induced volume charge density follows the field with a certain delay. The resulting volume force produces a torque on the liquid and the appearance of rotation of the fluid around a central axis.

\subsection{Basic electric field}

We consider the $x$ - and $y$-axes along the gaps and the $z$-axis normal to the electrode plane. 
In the lowest order, the electric potential satisfies Laplace's equation with the boundary condition at the plane $z=0$

$$
\phi=V_{s}(x, y, t) \begin{cases}V_{0} \cos (\omega t), & x>0, y>0, \\ -V_{0} \sin (\omega t), & x<0, y>0, \\ -V_{0} \cos (\omega t), & x<0, y<0, \\ V_{0} \sin (\omega t), & x>0, y<0\end{cases}
$$

and with the potential vanishing far from the electrodes

$$
\phi \rightarrow 0 \quad(z \rightarrow \infty) .
$$

This problem can be posed in terms of the complex potential amplitude, that also satisfies Laplace's equation, with the boundary condition

$$
\phi(z=0)=V_{s}= \begin{cases}V_{0}, & x>0, y>0, \\ V_{0} \mathrm{i}, & x<0, y>0, \\ -V_{0}, & x<0, y<0, \\ -V_{0} \mathrm{i}, & x>0, y<0 .\end{cases}
$$

The surface voltage can be written in a simpler form as follows

$$
V_{s}=\frac{V_{0}(1-\mathrm{i})}{2} \operatorname{sgn}(x)+\frac{V_{0}(1+\mathrm{i})}{2} \operatorname{sgn}(y),
$$

which corresponds to the symbolic equation:

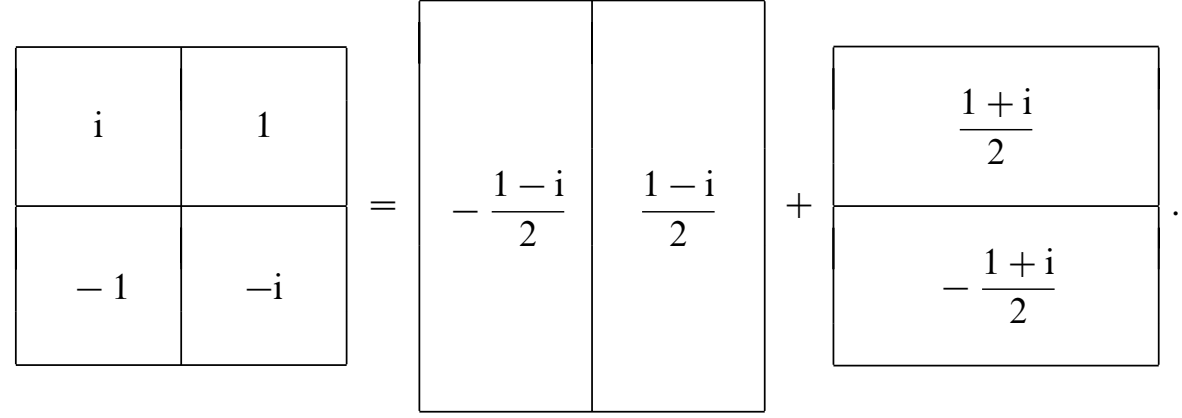

Each term of (38) is exactly the surface potential for the two-dimensional case that was described in the previous section. In this way, we can write the electric potential in the bulk as a sum of two two-dimensional solutions. These solutions are shifted $90^{\circ}$ in space and in time, producing the rotating potential. Writing the angles in terms of Cartesian coordinates we have

$$
\phi=\frac{V_{0}(1-\mathrm{i})}{\pi} \arctan \left(\frac{x}{z}\right)+\frac{V_{0}(1+\mathrm{i})}{\pi} \arctan \left(\frac{y}{z}\right) .
$$

The total complex amplitude for the electric field can also be expressed as a superposition of two fields

$$
\boldsymbol{E}_{0}=\frac{V_{0}(1-\mathrm{i})}{\pi}\left(\frac{-z \boldsymbol{u}_{x}+x \boldsymbol{u}_{z}}{x^{2}+z^{2}}\right)+\frac{V_{0}(1+\mathrm{i})}{\pi}\left(\frac{-z \boldsymbol{u}_{y}+y \boldsymbol{u}_{z}}{y^{2}+z^{2}}\right) .
$$


The direction of the electric field (in the time domain) rotates, although not uniformly. This field can be written equivalently as

$$
\boldsymbol{E}_{0}=\frac{1-\mathrm{i}}{\sqrt{2}} \boldsymbol{E}_{a}+\frac{1+\mathrm{i}}{\sqrt{2}} \boldsymbol{E}_{b},
$$

with $\boldsymbol{E}_{a}$ and $\boldsymbol{E}_{b}$ purely real, multiplied by complex coefficient of unit modulus. The first component is

$$
\boldsymbol{E}_{a}=\frac{V_{0} \sqrt{2}}{\pi}\left(\frac{-z \boldsymbol{u}_{x}+x \boldsymbol{u}_{z}}{x^{2}+z^{2}}\right)
$$

which is identical to the two-dimensional field given in (18) except for the factor $\sqrt{2}$.

\subsection{The case of a vertical gradient}

We now consider the combined action of the previous electric field with an imposed uniform vertical temperature gradient, $\nabla T=T^{\prime} \boldsymbol{u}_{z}$.

The volume force

$$
\langle\boldsymbol{f}\rangle=\frac{1}{2} \operatorname{Re}\left(\frac{\varepsilon^{0}(\alpha-\beta)}{1+\mathrm{i} \omega \tau}\left(\nabla T \cdot \boldsymbol{E}_{0}\right) \boldsymbol{E}_{0}^{*}\right)-\frac{\varepsilon^{0} \alpha}{4}\left(\boldsymbol{E}_{0} \cdot \boldsymbol{E}_{0}^{*}\right) \nabla T,
$$

and is composed of a Coulomb part and a dielectric one. Both can be decomposed into four terms, according to the components, $\boldsymbol{E}_{a}$ and $\boldsymbol{E}_{b}$, of the electric field involved

$$
\boldsymbol{f}=\boldsymbol{f}_{a a}+\boldsymbol{f}_{a b}+\boldsymbol{f}_{b a}+\boldsymbol{f}_{b b} .
$$

The first and last terms produce purely two-dimensional motions, as described in a previous section: $f_{a a}$ generates a two-dimensional motion with components in the $(x, z)$-plane, and $f_{b b}$ in the $(y, z)$-plane. The velocity expressions for these lateral motions are the same as in (27) and (28) multiplied by a factor 2. In what follows, we are mostly concerned with the cross-terms, $\boldsymbol{f}_{a b}$ and $\boldsymbol{f}_{b a}$, which produce net rotation.

The cross-terms for the dielectric force cancel each other

$$
\boldsymbol{f}_{d}=-\frac{\varepsilon^{0} \alpha}{4} \nabla T\left(\mathrm{i} \boldsymbol{E}_{b} \cdot \boldsymbol{E}_{a}^{*}-\mathrm{i} \boldsymbol{E}_{a} \cdot \boldsymbol{E}_{b}^{*}\right)=0 .
$$

The only new terms appear in the Coulomb force:

$$
\boldsymbol{f}_{a b}+\boldsymbol{f}_{b a}=\frac{\varepsilon^{0}(\alpha-\beta) \omega \tau}{\left(1+\omega^{2} \tau^{2}\right)}\left(\left(\nabla T \cdot \boldsymbol{E}_{a}\right) \boldsymbol{E}_{b}-\left(\nabla T \cdot \boldsymbol{E}_{b}\right) \boldsymbol{E}_{a}\right) .
$$

Even before calculating the spatial dependence of the coupled volume force density, we can establish the dependence on the frequency as

$$
\boldsymbol{f}_{a b}+\boldsymbol{f}_{b a}=\frac{\omega \tau}{1+\omega^{2} \tau^{2}} \boldsymbol{C} .
$$

This term vanishes for low and high frequencies, with a maximum at the charge relaxation frequency $\omega=\sigma^{0} / \varepsilon^{0}$. Contrary to the previous two-dimensional fluid flows, this three-dimensional case does not change direction with frequency.

The spatial dependence of the coupled force density is

$$
\boldsymbol{f}_{c}=\frac{A z\left(-y \boldsymbol{u}_{x}+x \boldsymbol{u}_{y}\right)}{\left(x^{2}+z^{2}\right)\left(y^{2}+z^{2}\right)}, \quad A=-\frac{V_{0}^{2} T^{\prime}(\alpha-\beta) \varepsilon^{0} \omega \tau}{\pi^{2}\left(1+\omega^{2} \tau^{2}\right)},
$$

or, using spherical coordinates, 


$$
\boldsymbol{f}_{c}=\frac{A \cos \theta \sin \theta}{r^{2}\left(\cos ^{2} \theta+\sin ^{2} \theta \cos ^{2} \varphi\right)\left(\cos ^{2} \theta+\sin ^{2} \theta \sin ^{2} \varphi\right)} \boldsymbol{u}_{\varphi} .
$$

This force is directed along the azimuthal direction, causing rotation.

The dependence on the angular coordinate $\varphi$ is periodic with a period of length $\pi / 2$, repeating each quadrant, and therefore it can be written as a Fourier series,

$$
f_{c}=\sum_{n=-\infty}^{\infty} c_{n} \mathrm{e}^{\mathrm{i} 4 n \varphi} .
$$

A somewhat lengthy calculation leads to the coefficients

$$
c_{n}=\frac{2 A \sin \theta}{r^{2}\left(1+\cos ^{2} \theta\right)}\left(\tan \left(\frac{\theta}{2}\right)\right)^{|n|} .
$$

Only the zero-order term produces net rotation. The component of this mode is obtained by averaging in $\varphi$ over a period

$$
\frac{1}{2 \pi} \int_{0}^{2 \pi} f_{c} \mathrm{~d} \varphi=c_{0}=\frac{2 A \sin \theta}{r^{2}\left(1+\cos ^{2} \theta\right)} .
$$

Multiplying the Navier-Stokes equations by $\boldsymbol{u}_{\varphi}$ and averaging in $\varphi$, we obtain an equation for the mean azimuthal velocity

$$
\frac{1}{r} \frac{\partial^{2}}{\partial r^{2}}\left(r\left\langle v_{\varphi}\right\rangle\right)+\frac{1}{r^{2}} \frac{\partial}{\partial \theta}\left(\frac{1}{\sin \theta} \frac{\partial}{\partial \theta}\left(\sin \theta\left\langle v_{\varphi}\right\rangle\right)\right)=-\frac{2 A \sin \theta}{\eta r^{2}\left(1+\cos ^{2} \theta\right)} .
$$

As in the previous sections, the $r^{-2}$-dependence of the force dictates the functional form for the self-similar velocity solution. We assume a functional form independent of $r$ as $\left\langle v_{\varphi}\right\rangle=U(\theta) A / \eta$, which reduces the problem to an ordinary differential equation,

$$
\frac{\mathrm{d}}{\mathrm{d} \theta}\left(\frac{1}{\sin \theta} \frac{\mathrm{d}}{\mathrm{d} \theta}(\sin \theta U)\right)=-\frac{2 \sin \theta}{1+\cos ^{2} \theta} .
$$

The solution for this equation that satisfies the b.c. of zero velocity at $\theta=0$ and at $\theta=\pi / 2$ is

$$
U=\cot \theta\left(\frac{\pi}{2}-2 \arctan (\cos \theta)-\ln (2)\right)+\frac{\ln \left(1+\cos ^{2} \theta\right)}{\sin \theta} .
$$

This function is always positive, and goes from zero at the axis to zero at the electrodes, reaching maximum speed at $\theta=0.961$ with a value $U_{\max }=0.232$ (see figure 4).

\section{Comparison with experiments}

The electrothermal fluid flows were observed on micro-electrode structures subjected to a.c. signals with potential amplitudes between 0 and $10 \mathrm{~V}(20 \mathrm{~V}$ peak to peak) and frequencies between 0.1 and $20 \mathrm{MHz}$. Two types of microelectrode design were fabricated on planar glass slides using photolithography: (a) two parallel electrodes separated by constant gap and $(b)$ four hyperbolic electrodes (the dimensions can be seen in figure 5). The pair of electrodes was subjected to a single a.c. signal. The four hyperbolic electrodes were driven by a four-phase a.c. signal, with the voltage on consecutive electrodes phase-shifted by $90^{\circ}$ in order to generate a rotating electric field. 


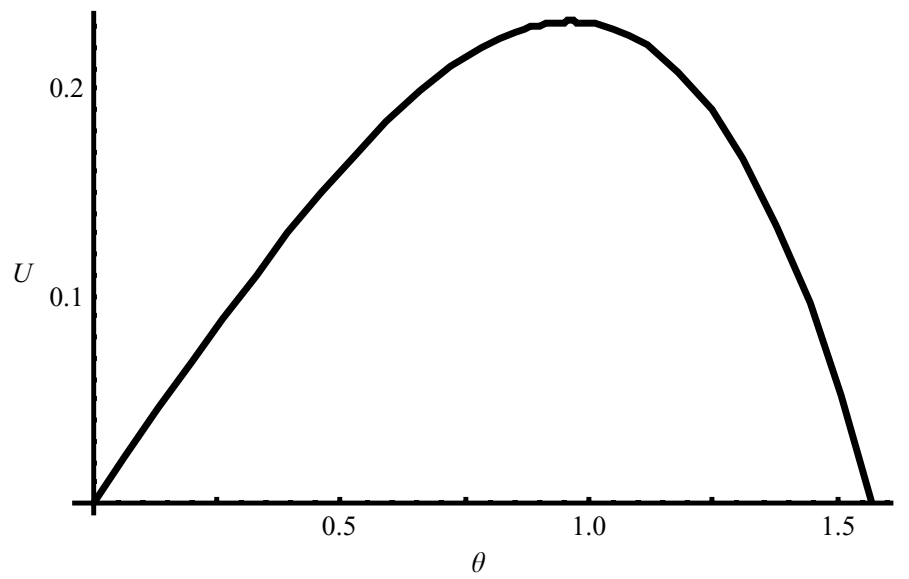

FIgURE 4. Plot of the velocity function $U$, (equation (55)), with polar angle $\theta$.

(a)

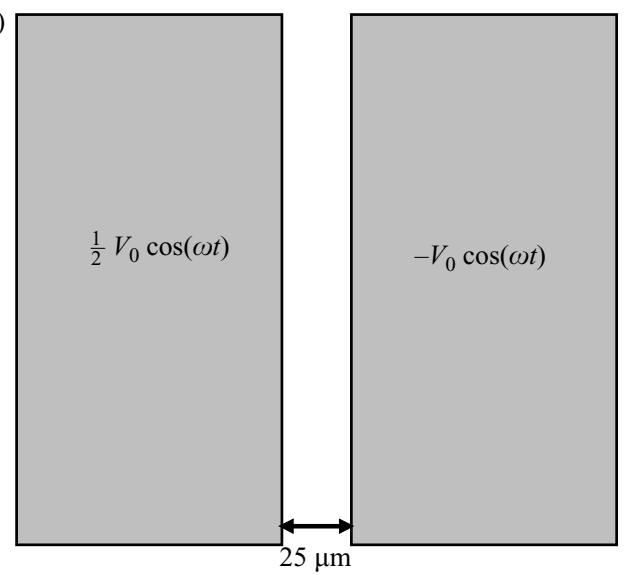

(b)

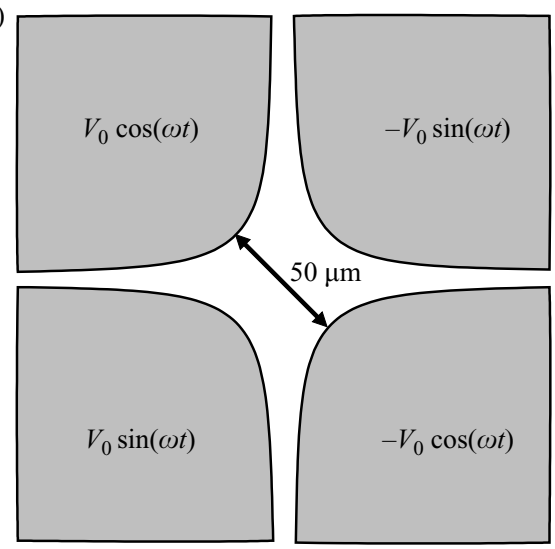

FIGURE 5. Diagram showing the two designs of electrode use in the experiments, with the voltage sequences used to drive the fluid.

A squared glass chamber was constructed around the electrodes and filled with a $\mathrm{KCl}$ solution of conductivity $\sigma=2.5 \times 10^{-3} \mathrm{~S} \mathrm{~m}^{-1}$. Fluorescent latex particles, $500 \mathrm{~nm}$ in diameter, were suspended in the $\mathrm{KCl}$ solution and used as tracers. The electrodes were observed and illuminated using epi-fluorescence microscopy with band-pass filters which allowed only light of a given wavelength to reach the sample. The fluid velocity was measured using particle-tracking velocimetry. Stacks of images taken from video were handled using free software from ImageJ (http://rsb.info.nih.gov/ij/).

In both types of electrode, the incoming light from the microscope was enough to heat the electrodes. A clear indication of this was the observation of natural convection for a chamber with a height of $1 \mathrm{~mm}$. In this chamber, the fluid was observed to move when the spot of incoming light was on the electrodes with no applied electric field. At the level of the electrodes, the fluid flowed towards the light spot as expected by natural convection. However, when the spot of light was on the glass, no observable fluid flow occurred. The conclusion is, therefore, that the light is heating the electrodes much more than the glass or the aqueous solution. For chambers with smaller heights, gravity could not overcome the viscous friction 


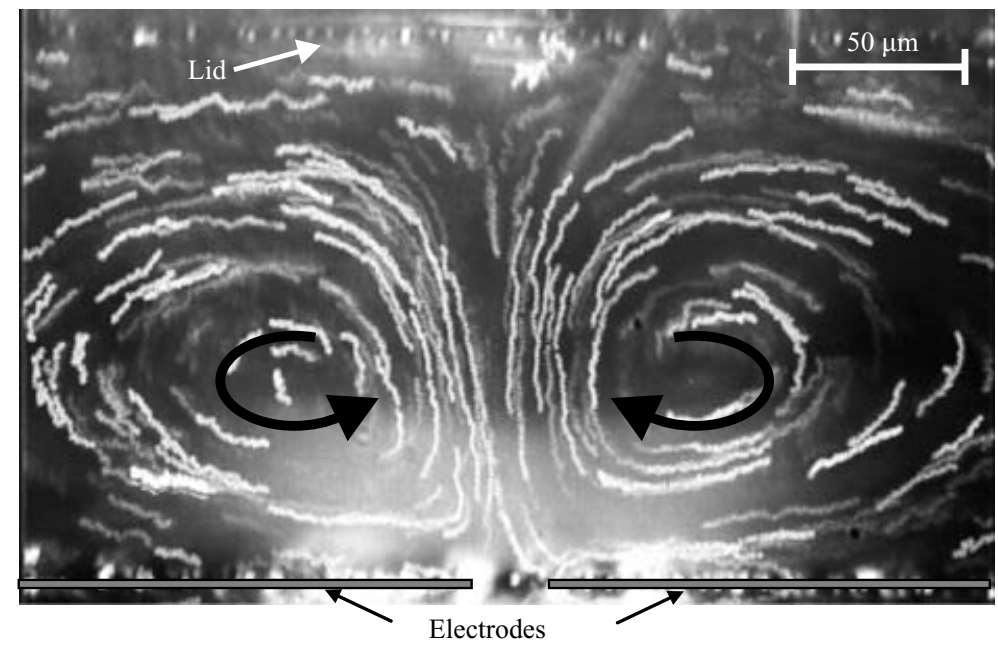

FIGURE 6. Horizontal view of two counter-rotating rolls on top of two coplanar electrodes at $V_{0}=7 \mathrm{~V}$ and $f=1 \mathrm{MHz}$. Image of particle tracks obtained by superimposing successive video frames.

and no observable motion was detected without electric fields. However, with the application of electric fields under illumination, fluid flow was observed in chambers with heights smaller than $200 \mu \mathrm{m}$. The buoyancy force becomes less important as the typical system length decreases. As discussed by Castellanos et al. (2003), a transition between buoyancy and electrothermal fluid flows is expected to occur for typical system lengths around $300 \mu \mathrm{m}$.

The magnitude of the incident light could be altered by inserting a filter into the light path. The light intensity was measured at different positions in the area of view using a photodiode. With a filter inserted into the light path, the light intensity decreased by a factor $3.62 \pm 0.15$. If the flow is electrothermal in origin, i.e. if the conductivity and permittivity gradients occur because of light-induced heating of the liquid, then the velocity of the liquid is expected to change by the ratio of light intensity with and without the filter.

\subsection{Experiments with a pair of symmetric electrodes}

Observations of fluid flows under conditions similar to the two-dimensional problems previously analysed have been reported in Green et al. (2000). Fluid flow was observed when the electrodes were subjected to voltages around $10 \mathrm{~V}$ and frequencies around $1 \mathrm{MHz}$ under strong illumination. The observed fluid flow pattern agrees well with the prediction from the electrothermal theory if there is a vertical gradient of temperature, generated by the light (Green et al. 2001). The experimental fluid flow changed direction at a certain frequency and the fluid velocity amplitude varied with frequency as predicted. The two-dimensional analytical solutions of the present work compare well with the numerical and experimental results.

In the present work, the fluid was observed from the side using a horizontal microscope. Figure 6 shows a superimposition of several images showing the experimental streamlines, for a voltage amplitude of $V_{0}=7 \mathrm{~V}$ and frequency of $f=1 \mathrm{MHz}$. The lid of the device was at a height of $160 \mu \mathrm{m}$. The fluid velocity was measured at a height of $77 \pm 4 \mu \mathrm{m}$ above the electrode gap. It was found that the flow velocity was reduced by a factor of $3.5 \pm 0.5$ when the incident light was reduced 


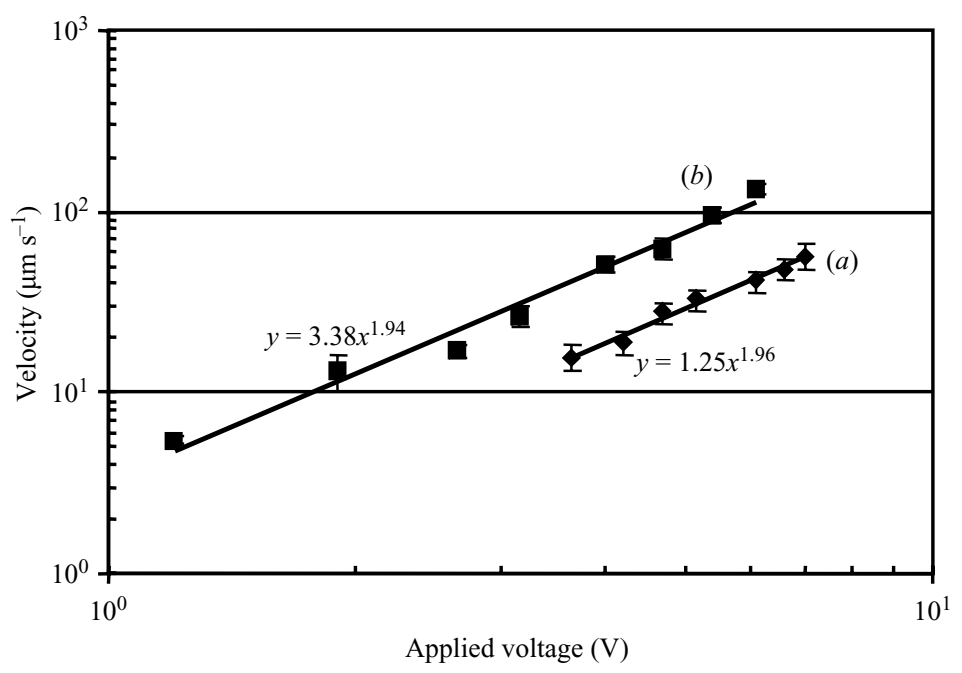

FigURE 7. Best-fit power law of velocity against applied voltage at $f=1 \mathrm{MHz}$ : $(a)$ two coplanar electrodes subjected to a single phase signal, and $(b)$ four coplanar electrodes subjected to a four-phase signal.

with a filter. This factor corresponds to the ratio of light intensities with and without the filter, and indicates that the flow is electrothermal.

The velocity as a function of voltage amplitude $V_{0}$ at the same position and frequency is shown in figure 7. The best-fit power curve is given by $u=1.25 V_{0}^{1.96} \mu \mathrm{m} \mathrm{s}^{-1}$ (with $V_{0}$ in volts), close to the expected power law $u \propto V_{0}^{2}$. At this height of $77 \mu \mathrm{m}$, dielectrophoresis on the tracking particles should be negligible (Ramos et al. 1998). A maximum estimated dielectrophoretic velocity is around $0.01 V_{0}^{2} \mu \mathrm{m} \mathrm{s}^{-1}$, much smaller than the measured velocities.

In previous experiments (Green et al. 2000), with a voltage of $V_{0}=10 \mathrm{~V}$ the velocity was found to be $80 \mu \mathrm{m} \mathrm{s}^{-1}$. In the present work, we measured a value of $110 \mu \mathrm{m} \mathrm{s}^{-1}$ for the same voltage. This discrepancy could be explained by a change in the vertical temperature gradient, by a factor 1.37 times greater than in the previous experiments.

\subsection{Experiments on electrothermal rotation}

Figure 8 shows particle tracks at a plane $40 \mu \mathrm{m}$ up from the electrodes for two cases: $(a)$ with maximum incident light, and $(b)$ with the light intensity reduced by a factor 3.6 with a filter. The electrodes were driven with a four-phase sine wave with a voltage amplitude of $V_{0}=6 \mathrm{~V}$ and a frequency of $f=3 \mathrm{MHz}$. The particle trajectories demonstrate that the liquid rotates when subjected to a rotating electric field under strong illumination. When the direction of the rotating field was changed, the liquid changed direction accordingly. Because there is more light in case $(a)$, the particle track lengths are greater than in case $(b)$ for the same time interval. The ratio between measured velocities at selected points in case $(a)$ and in case $(b)$ is $3.4 \pm 0.2$, in agreement with the ratio of light intensities.

Figure 7 shows the velocity as a function of voltage, measured at a radius of $100 \mu \mathrm{m}$ from the centre of rotation, a height of $30 \mu \mathrm{m}$ above the electrodes, and a frequency of $1 \mathrm{MHz}$. The best-fit power curve is given by $u=3.38 V_{0}^{1.94} \mu \mathrm{m} \mathrm{s}^{-1}$, again close to the expected $V_{0}^{2}$ power law. At this position, the fluid speed was 
(a)

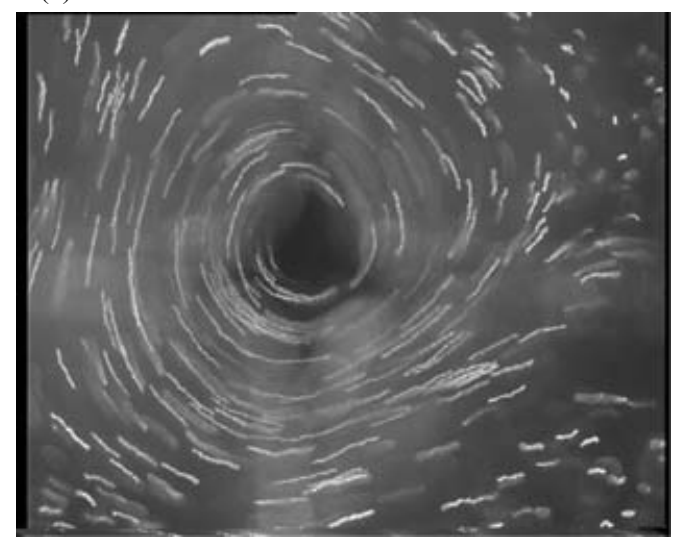

(b)

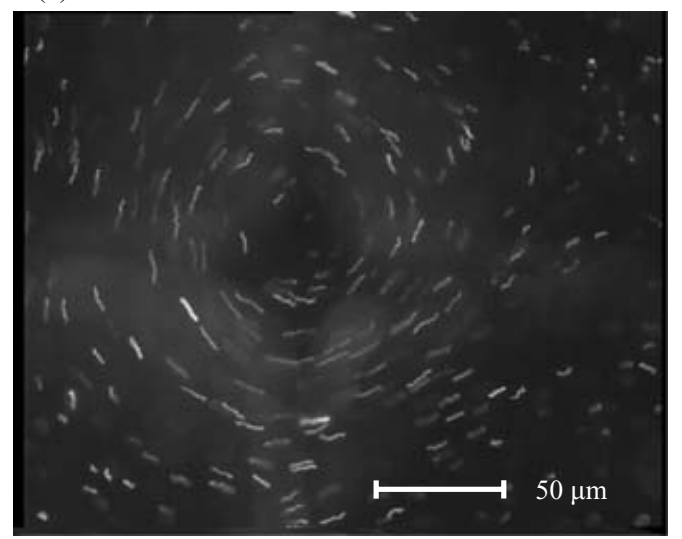

FIGURE 8. Top view of a rotating aqueous solution placed on top of four coplanar electrodes. Image of particle tracks obtained by superimposing successive video frames in a time interval of $0.4 \mathrm{~s}$ : (a) maximum light intensity, and $(b)$ light intensity reduced by filter.

$96 \pm 9 \mu \mathrm{m} \mathrm{s}^{-1}$ at $V_{0}=5.4 \mathrm{~V}$ and $1 \mathrm{MHz}$. In order to obtain this velocity using the

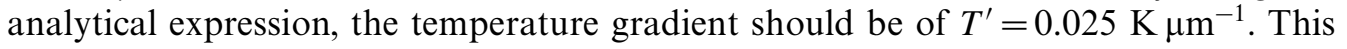
gradient of temperature is 1.2 times greater than the gradient estimated in previous experiments (Green et al. 2000, 2001).

As predicted by the three-dimensional analytical solution, the rotating fluid flow was significant over a certain frequency range and did not change direction with frequency. Figure 9 shows particle tracks from a top view at $V_{0}=5.8 \mathrm{~V}$ and four different frequencies: $0.2,0.5,2$ and $5 \mathrm{MHz}$. The fluid flow behaves differently at frequencies above and below $1 \mathrm{MHz}$. Above $1 \mathrm{MHz}$, the flow patterns are more circular, while below $1 \mathrm{MHz}$ they look like spirals. According to the theory, the flow is the sum of two superimposed characteristic fluid flows: $(a)$ a rotating flow with maximum rotation at $f=0.568 \mathrm{MHz}$ as given by $\omega=\sigma / \varepsilon$; and $(b)$ two superimposed two-dimensional flows with velocity components in the $(x, z)$ - and $(y, z)$-planes, and with a characteristic frequency of $1.27 \mathrm{MHz}$ as given by $\omega_{c}=(\sigma / \varepsilon) \sqrt{-\beta / \alpha}$. At this frequency, the two-dimensional flows disappear because Coulomb and dielectric forces cancel each other. The ratio between two-dimensional and rotational velocities is proportional to

$$
\frac{v_{2 D}}{v_{\text {rot }}} \propto \frac{|\beta-| \alpha\left|(\omega \tau)^{2}\right|}{(\beta+|\alpha|) \omega \tau} .
$$

This ratio is zero for the transition frequency $\omega_{c}$. For very high and very low frequencies, the two-dimensional motions dominate. There is an interval of frequencies around $\omega_{c}$ where the rotational motion is dominant. For $\omega \ll \omega_{c}$, the two-dimensional motion is proportional to $\beta$ whereas for $\omega \gg \omega_{c}$, the two-dimensional motion is proportional to $|\alpha| \sim|\beta| / 5$. Therefore, the frequency range where rotation is dominant is greater for $\omega>\omega_{c}$ than for $\omega<\omega_{c}$. This can explain why the spiral-like paths observed at $f<1 \mathrm{MHz}$ change to more circular paths at $f>1 \mathrm{MHz}$, since the twodimensional motions are relatively less important at these frequencies.

Figure 10 is a plot of the measured angular velocity as a function of frequency at constant voltage amplitude $V_{0}=5.8 \mathrm{~V}$. The angular velocity was measured from the particle tracks at different angles about the origin, at a radius of $85 \pm 25 \mu \mathrm{m}$ 
(a)

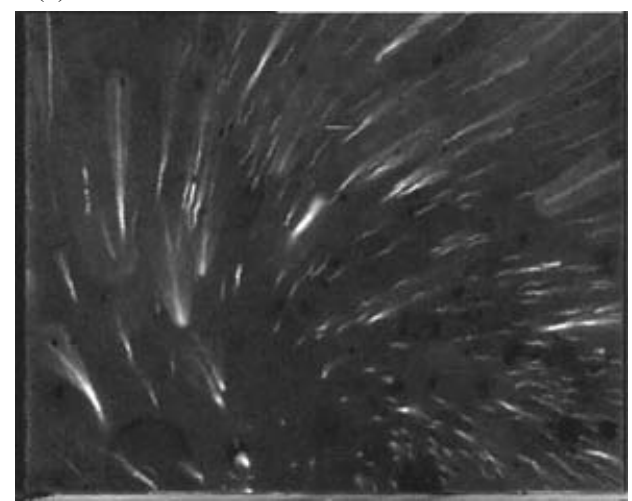

(c)

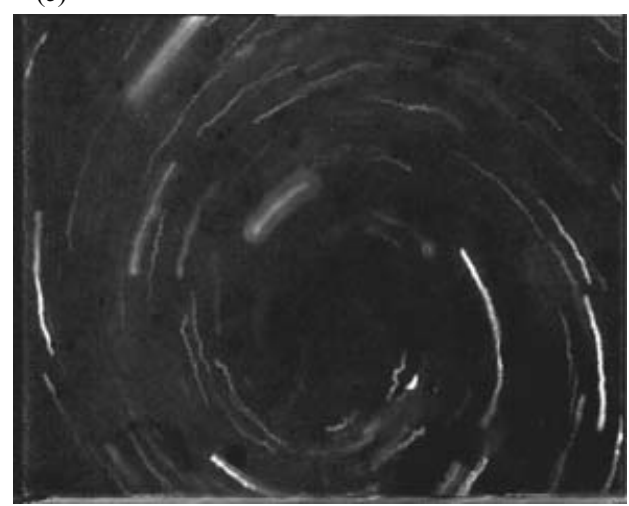

(b)

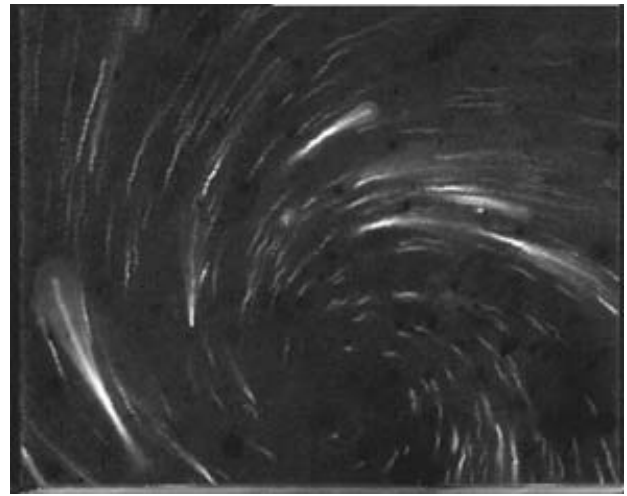

$(d)$

Figure 9. Top view of a rotating aqueous solution on top of four coplanar electrodes. The applied four-phase signals have an amplitude of $5.8 \mathrm{~V}$ and different frequencies: (a) $0.2 \mathrm{MHz}$, (b) $0.5 \mathrm{MHz},(c) 2 \mathrm{MHz}$ and (d) $5 \mathrm{MHz}$.

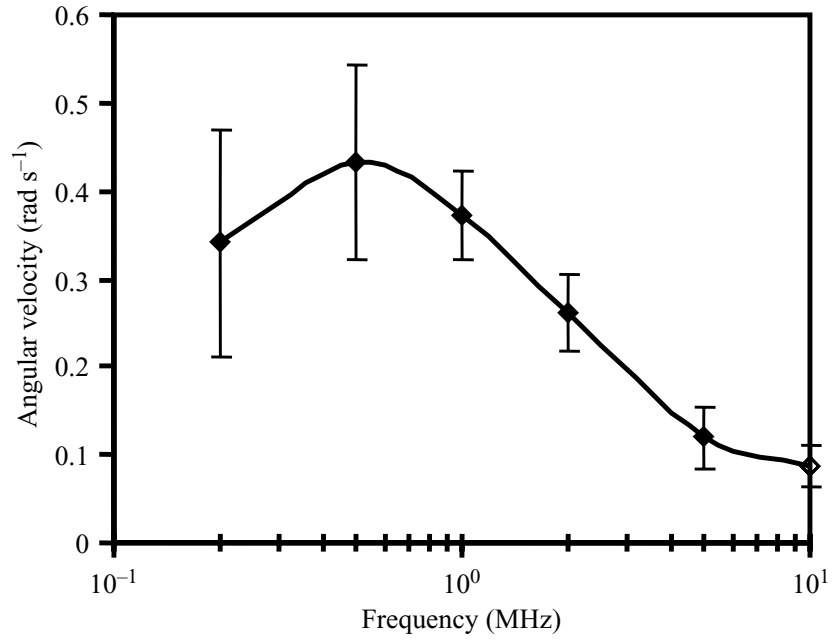

FIGURE 10. Angular velocity as a function of frequency.

from the centre, and at a height of $60 \mu \mathrm{m}$. The plotted data are the averages of these points. The error bars are given by the standard deviation. The large errors at $0.2 \mathrm{MHz}$ are an indication of the difficulty in assigning a rotatory velocity in 
this case (see figure 9). Theoretically, the maximum angular velocity is obtained at $f=\sigma^{0} / 2 \pi \varepsilon^{0}=0.568 \mathrm{MHz}$, in accordance with the experiments within experimental errors. The theoretical expression for the angular velocity at a given height $h$ from the electrodes is given by

$$
\Omega(\theta)=\frac{A}{h \eta} U(\theta) \cot \theta .
$$

The illumination intensity was different from the previous experiments and the measured angular velocities would be obtained if the temperature gradient is around four times smaller than for the data shown in figure 7.

\section{Conclusions}

Self-similar solutions for the fluid flow induced by the combination of electric fields and externally imposed gradients of temperature in microelectrode structures have been presented. Two kinds of problem were considered: $(a)$ the two-dimensional motion of an aqueous solution placed on top of two coplanar parallel electrodes subjected to an a.c. potential difference when there is either a vertical or a horizontal gradient of temperature; and $(b)$ the three-dimensional motion of an aqueous solution lying on four coplanar hyperbolic electrodes subjected to a four-phase a.c. signal, generating a rotating electric field.

Experiments were performed using aqueous solutions of $\mathrm{KCl}$ placed on top of two or four coplanar microelectrodes and the results compared with the analytical solutions. The temperature field was imposed using the light from the microscope. Indications of this were the observation of natural convection for devices with heights around $1 \mathrm{~mm}$ and that the observed electrothermal fluid velocities were proportional to the light intensity. The device with two coplanar parallel electrodes produced two-dimensional rolls of fluid flow. The device with four hyperbolic electrodes demonstrated that the liquid rotates when subjected to a rotating electric field under strong illumination. The experiments showed a good agreement with the expected trends from the theoretical solutions.

The combination of electric fields and heating with light at localized points could be of interest in the design of micro-pumps and for local mixing in the lab-on-a-chip technology. An advantage of this way of generating flow in saline solutions is that the maximum flow velocity is almost independent of the conductivity of the solution. In order to obtain the best efficiency, the frequency of the applied signal should be increased linearly with conductivity.

This work has been supported by the Spanish MCyT, under contract BFM200301739 .

\section{REFERENCES}

Ajdari, A. 2000 Pumping liquids using asymmetric electrode arrays. Phys. Rev. E 61, R45-R48.

ARnold, W. M. 2001 Positioning and levitation media for the separation of biological cells. IEEE Trans. Ind. Applics 37, 1468-1475.

BazANT, M. Z. \& SQuires, T. M. 2004 Induced-charge electrokinetic phenomena: theory and microfluidic applications. Phys. Rev. Lett. 92, 066101.

Bazant, M. Z., Thornton, K. \& Ajdari, A. 2004 Diffuse-charge dynamics in electrochemical systems. Phys. Rev. E 70, 021506.

Castellanos, A. 2003 Entropy production and the temperature equation in electrohydrodynamics. IEEE Trans. Dielectrics Elect. Insul. 10, 22-26. 
Castellanos, A., Ramos, A., González, A., Green, N. G. \& Morgan, H. 2003 Electrohydrodynamics and dielectrophoresis in microsystems: scaling laws. J. Phys. D: Appl. Phys. 36, 2584-2597.

Chen, H.-H., Lin, H., Lele, S. K. \& Santiago, J. G. 2005 Convective and absolute electrokinetic instability with conductivity gradients. J. Fluid Mech. 524, 263-303.

Gimsa, J., EPPMANN, P. \& PrüGER, B. 1997 Introducing phase analysis light scattering for dielectric characterization: measurement of travelling-wave pumping. Biophys. J. 73, 3309-3316.

Green, N. G., Ramos, A., González, A., Castellanos, A. \& Morgan, H. 2000 Electric field induced fluid flow on microelectrodes: the effect of illumination. J. Phys. D: Appl. Phys. 33, L13-L17.

Green, N. G., Ramos, A., González, A., Castellanos, A. \& Morgan, H. 2001 Electrothermally induced fluid flow on microelectrodes. J. Electrost. 53, 71-87.

Haus, H. A. \& Melcher, J. R. 1989 Electromagnetic Fields and Energy. Prentice-Hall.

Levich, V. G. 1962 Physicochemical Hydrodynamics. Prentice-Hall.

Lide, D. R. (ed.) 1994 CRC Handbook of Chemistry and Physics, 74th edn. CRC Press, London.

Melcher, J. R. Continuum Electromechanics. 1981 The MIT Press, Cambridge, MA.

Melcher, J. R. \& Firebaugh, M. S. 1967 Traveling-wave bulk electroconvection induced across a temperature gradient. Phys. Fluids 10, 1178-1185.

Melcher, J. R. \& TAYLoR, G. I. 1969 Electrohydrodynamics: a review of the role of interfacial shear stresses. Annu. Rev. Fluid Mech. 1, 111-146.

Müller, T., Arnold, W. M., Schnelle, T., Hagedorn, R., Fuhr, G. \& Zimmermann, U. 1993 A traveling-wave micropump for aqueous solutions: comparison of $1 \mathrm{~g}$ and $\mu \mathrm{g}$ results. Electrophoresis 14, 764-772.

Müller, T., Gerardino, A., Schnelle, Th., Shirley, S. G., Bordoni, F., De Gasperis, G., Leoni, R. \& FuHR, G. 1996 Trapping of micrometre and sub-micrometre particles by high-frequency electrical fields and hydrodynamic forces. J. Phys. D: Appl. Phys. 29, 340-349.

Putnam, S. A. \& CAhill, D. G. 2005 Transport of nanoscale latex spheres in a temperature gradient. Langmuir 21, 5317-5323.

Ramos, A., Morgan, H., Green, N. G. \& Castellanos, A. 1998 AC electrokinetics: a review of forces in microelectrode structures. J. Phys. D: Appl. Phys. 31, 2338-2353.

Ramos, A., Morgan, H., Green, N. G. \& Castellanos, A. 1999 AC electric-field-induced fluid flow in microelectrodes. J. Colloid Interface Sci. 217, 420-422.

SAville, D. A. 1997 Electrohydrodynamics: the Taylor-Melcher leaky dielectric model. Annu. Rev. Fluid Mech. 29, 27-64.

Schnelle, T., Müller, T., Reichle, C. \& Fuhr, G. 2000 Combined dielectrophoretic field cages and laser tweezers for electrorotation. Appl. Phys. B 70, 267-274.

Tritton, D. J. 1977 Physical Fluid Dynamics. Van Nostrand Reinhold.

Wang, D., Sigurdson, M. \& Meinhart, C. D. 2005 Experimental analysis of particle and fluid motion in a.c. electrokinetics. Exps. Fluids 38, 1-10. 